\title{
EI Día del Biólogo y su significado en el desarrollo de las Ciencias Biológicas en el Perú
}

\author{
Dr. Pedro G. Aguilar Fernández*
}

Distinguidos miembros de la Mesa Directiva, colegas y futuros colegas, damas y caballeros:

Es muy grato poder conmemorar con ustedes los 53 años del establecimiento del Día del Biólogo, cuando la Facultad de Ciencias de San Marcos ha cumplido su $139 .^{\circ}$ aniversario.

Quien les habla pertenece a la Promoción de Ciencias Biológicas de San Marcos 1948, denominada "Javier Ortiz de LA Puente", en honor a uno de los más sobresalientes biólogos orientados a la zoología, que falleció muy joven, pero que a su paso por las aulas sanmarquinas, como estudiante y como docente, dejó profundas huellas de su bonhomía. Nuestra promoción, desde su inicio en 1945, como corresponde a toda la juventud que desea afianzar la bases de su preparación para la vida, demostró su entusiasmo, su dedicación y su agradecimiento por las enseñanzas recibidas, pero al mismo tiempo se unió al clamor estudiantil existente, desde anteriores promociones, para mejorar la plana docente, para modernizar los programas de estudio, el contenido de las prácticas, las facilidades de laboratorio, las salidas al campo, la recolección y preparación de material para enseñanza e investigación.

Referiré algunos hechos notables en la historia de la enseñanza universitaria de las ciencias biológicas en el Perú.

Bólogo UNMSM, Promoción 1948, docente UNMSM y UNALM, Lína

Discurso de Orden por el Dia del Biólogo

Viemes 1." diciembre 2000, 12:00 h

Facultad do Ciencias Bioboicas UNMSM
La fundación de la Facultad de Ciencias en la Universidad Nacional Mayor de San Marcos y en las otras universidades Ilamadas menores

El 28 de agosto de 1861 el gobierno peruano había dispuesto la creación de la Facultad de Matemáticas y Ciencias Naturales en la Universidad de San Marcos, comprendiendo cuatro áreas: (1) Matemáticas; (2) Física (incluyendo Astronomía); (3) Química; (4) Historia Natural (Mineralogía, Geología, Botánica y Zoología), área en la que el distinguido naturalista italiano don ANTONIO RAIMONDI fue profesor fundador. El 15 de marzo de 1866 se independizó de la Facultad de Letras y Jurisprudencia. Su primer decano, designado por el gobierno, fue ANTONIO RaImond, quien renunció al cabo de un año, pues para él era mucho más importante continuar el estudio de la naturaleza peruana que hacer vida administrativa y docente.

Actualmente, cada una de esas áreas, Biología, Física, Geología, Matemática, Química, constituye facultad independiente, con departamentos académicos especializados que demuestran el desarrollo alcanzado por estas ciencias.

Por entonces, en 1866, las otras universidades nacionales, llamadas menores, eran las de Arequipa, Cusco y Trujillo.

La Universidad Nacional San Antonio Abad del Cusco se fundó el $10^{\circ}$ de marzo de 1696. Se inició la enseñanza de ciencias biológicas en 1911. Con el Museo de Plantas creado por el Dr. Fortunato Herrera en $1925 \mathrm{se}$ fortaleció la Escuela Botánica, cuyo mejor 
representante ha sido el Dr. CÉSAR VARGAS. En 1950 se creó el Museo de Historia Natural en el Cusco.

La Universidad Nacional de Trujillo fue fundada el 10 de mayo de 1824 e instalada el 12 de octubre de 1831. La enseñanza de las ciencias biológicas se inició en 1928, dentro de una Facultad de Ciencias Físicas y Naturales; se acentuó su desarrollo con la creación de los museos de Zoología en 1938, por don Juan Ormea; y el de Botánica en 1941, por don Nicolás Angulo. En 1962 se creó la Facultad de Ciencias Biológicas con tres currículos: Biología, Biología Pesquera y Microbiología.

La Universidad Nacional San Agustín de Arequipa se fundó el 11 de noviembre de 1828. Las ciencias biológicas se iniciaron en 1928 dentro de una Facultad de Ciencias Naturales. Actualmente existe la Facultad de Ciencias Biológicas y Agropecuarias con las carreras de Biología, Pesquería, Agronomía y Zootecnia.

\section{Don Antonio Raimondi y la Facultad de Ciencias de la UNMSM}

Don Antonio Raimond, el primer decano de esta Facultad de Ciencias, en gran parte fue un autodidacta que, en su tierra natal, desde los trece años con sus ahorros compraba obras de los naturalistas de la época como BUFFoN, visitaba museos de historia natural, jardines botánicos, colecciones de animales vivos; realizaba excursiones al campo, estudiando plantas, animales, rocas y minerales. Leer las obras de los viajeros geógrafos y naturalistas como BougainviLle y HumboldT despertó en Raimondi el más vivo deseo de visitar América del Sur, eligiendo al Perú como su destino.
Llegó al Perú el 28 de julio de 1850, a la edad de 24 años; no trajo ningún grado académico ni título profesional. Inició sus viajes de estudio en 1851. Dieciocho años después, en enero de 1869 el Congreso peruano autorizó la publicación de la obra raimondiana titulada El Perú. El primer tomo apareció en 1874, en 1876 el Tomo II, en 1880 el Tomo III, los que él mismo pudo dirigir. RAIMONDI falleció en el Perú, en San Pedro de Lloc (Pacasmayo) en octubre de 1890 , después de 40 años de muy esforzadas observaciones sobre el ambiente natural del Perú, superando todas las dificultades de la época. Su propósito inicial había sido dedicar unos diez años a recoger objetos de historia natural y notas en el mismo lugar. para regresar a Europa y allá publicar los resultados de sus estudios.

Después de la muerte de Antonio RaIMONDI, la Sociedad Geográfica de Lima, por encargo del gobierno peruano, publicó en 1902 el tomo IV; en 1911 el V y en 1913 el VI. También publicó sus hojas del "Mapa del Perú", que resultó fundamental hasta la aparición de las hojas elaboradas por el Instituto Geográfico Militar del Perú. En 1965, con el auspicio de la Universidad Nacional de Ingeniería, se llevó a cabo una reimpresión, o publicación facsimilar, en cinco tomos de su obra El Perú, que comprende Geografía, Geología, Mineralogía, Botánica, Zoología y Etnología.

Todo el material colectado por RAIMONDI fue cedido a la Escuela de Medicina, y comprendía 300 objetos de Etnología; 400 aves, mamíferos y reptiles; 1500 molusos terrestres y fluviátiles; 500 moluscos marinos; 4000 insectos; 2000 conchas, dientes y huesos fósiles; 20000 plantas secas; 300 frutos, cortezas, gomas y resinas, 3000 rocas y minerales. Posteriormente este material enriqueció las colecciones del Museo de Historia Natural de la Universidad de San Marcos, que se fundó en 1918. 
En este año 2000, el último del siglo XX, y en vísperas del siglo XXI, no debemos olvidar las palabras que RAIMONDI dirigió a la juventud peruana del siglo XIX, en 1874 , hace 126 años, en el Tomo I de su obra El Perú:

"Jóvenes peruanos: Confiado en mi entusiasmo he emprendido un arduo trabajo muy superior a mis fuerzas. Os pido, pues, vuestro concurso. Ayudadme. Dad tregua a la politica y consagraos a hacer conocer vuestro país y los inmensos recursos que tiene"

Tres acontecimientos importantes en la Facultad de Ciencias de San Marcos antes de 1930, con relación a las Ciencias Biológicas

\section{La publicación de la Revista de Cien- cias de la UNMSM}

Las primeras autoridades de la Facultad de Ciencias de San Marcos fueron principalmente matemáticos, químicos o médicos aficionados a las ciencias naturales; probablemente muy pocos con vocación absoluta a ellas. Sin embargo, la Revista de Ciencias, publicación fundada en 1897, salió ininterrumpidamente durante 69 años, hasta 1966, en que cambió de formato y perdió continuidad. Se incluyeron trabajos de profesores de la propia universidad o de otras universidades, peruanas o del extranjero, sobre plantas medicinales, flora y vegetación, fósiles, antropología, biología andina, insectos, aves o recursos naturales como el guano de islas. Hubo, pues, inquietud en desarrollar trabajos en Biología, pero no destacaron líneas propias de investigación institucional.

\section{La Fundación del Museo de Historia Natural de la UNMSM}

La fundación del Museo de Historia Natural fue el 28 de febrero de 1918. Inicialmente funcionó en los altos de la Facultad de Letras de la casona; su primer director fue Don CARlos Rospigliosi Vigil, doctor en Ciencias Na- turales y médico asimilado al ejército, donde alcanzó el grado de coronel. Desde 1914 era profesor de Zoología General. En 1918 y 1920 dirigió dos "Expediciones científicas universitarias", con botánicos, zoólogos, geólogos, geógrafos, físicos y también gente de letras, para ver aspectos históricos de las regiones. Sus colecciones se incorporaron al Museo.

\section{La incorporación de Augusto Weber- BAUER a San Marcos}

La participación del Dr. Augusto Weberbauer en la enseñanza de la Botánica en la Facultad de Farmacia se produjo en 1921. Este profesor había llegado al Perú el 11 de noviembre de 1901 a la edad de 30 años, e indesmayablemente viajó por el país para cumplir con su interés en el estudio de la vegetación andina, y terminar su Mapa Fitogeográfico de los Andes Peruanos en 1917, cuya publicación logró con sus propios medios, primero en alemán, en 1922; y al año siguiente, con la ayuda del gobierno peruano, en español.

El año 1922 Augusto Weberbauer sustentó una investigación acerca de "La estructura anatómica de las hojas y la altitud sobre el nivel del mar", como tesis para Doctor en Ciencias Biológicas en la Universidad de San Marcos, con el fin de revalidar su grado de Doctor (Dr. Phil.) obtenido en la Universidad de Berlín, en 1898.

Weberbauer, como profesor de Botánica Sistemática, fue además Director del Jardín Botánico de Lima, Botánico Asesor de la Estación Experimental Agrícola La Molina y colaborador en la enseñanza de la Botánica en la Escuela Nacional de Agricultura, con el ingeniero agrónomo belga JuLio GaUdron. Allí conservó su herbario que actualmente con el nombre Herbario Weberbauer se encuentra en Facultad de Ciencias de la Universidad Nacional Agraria La Molina. Así, Weberbauer constituyó el punto de partida de la Escuela Botánica Peruana en San Marcos. 


\section{Algunos hechos destacables entre los años 1930 y 1948}

Fue después del receso de San Marcos (1932 a 1935) y comienzos de los años 40, cuando se vio surgir la nueva corriente en las ciencias biológicas de San Marcos, tanto propugnada por los docentes jóvenes como apoyada por la inquietud estudianti]. Es justo recordar que ese impulso fue haciéndose realidad poco a poco, con la ayuda del decanoDARío ACEVEDo, profesor de Fisiología General, médico asimilado a la Policía Nacional, que ejerció el decanato durante 16 años consecutivos (1946 a 1962) y en años posteriores por el Decano Rafael Dávila Cuevas, climatólogo formado en la Facultad de Ciencias de San Marcos y especializado en Japón.

En 1936, el Museo de Historia Natural, nominado "Javier Prado" pasó a ocupar el local nuevo de la cuadra 12 de la avenida Arenales. Ese nombre fue dado en agradecimiento al rector que había otorgado todo el apoyo para su construcción. En 1937 el Museo inició la publicación de su Boletín, que se editó sin interrupción hasta 1946. El concepto de su fundador, Carlos Rospigliosi Vigil, era: "Un museo no es sólo lugar de exhibición, sino lugar de trabajo e investigación". Rospigliosi falleció en 1938; lo siguió en la Dirección del Museo el médico Carlos Morales Macedo, Profesor de Biología General. Después de 1946, hasta la fecha, han ejercido la dirección del MHN, Jehan Vellard, BerNARdo Boit, Ramón Ferreyra y en los últimos años, Hernando de Macedo, Emma Cerrate, Gerardo lamas, Hernán Ortega, Magda Chanco y Niels Valencia.

En el área de Botánica, en 1945, Luis Gamarra Hernández era el profesor principal de Botánica General, pero Augusto Weberbauer había afianzado su Escuela de Botánica Sistemática, tanto en Ciencias como en Farmacia y ya había jóvenes botánicos como Celia Albrizzio, Clara Canales, Pedro Coronado, Ramón Ferreyra, JuAna InFantes,
Luz Valcárcel y Octavio Velarde, que destacaban con firmeza y serenidad. También JuLIoLóPEZ-GuILLÉN (farmacéutico) y FERNANdo Cabieses (médico) fueron alumnos de Weberbauer.

El año 1945 apareció la obra cumbre de Weberbauer El mundo vegetal de los Andes peruanos, de 776 páginas ilustradas, dedicada a la memoria de Antonio Raimondi. Por ella, en 1947 mereció la condecoración La Orden del Sol del Perú en el grado de Comendador. Es oportuno reproducir las palabras del insigne historiador Jorge BASADRE en dicha ocasión:

"Su paciencia de hormiga llevó a WEBERBAUER a construir, fragmento tras fragmento, una obra gigante. Por su cuidado y escrupulosidad en los detalles de ella, parece que hizo suyas las palabras de GOETHE: Que CADA PASO SEA UNA META SIN DEJAR DE SER UN PASO. Resulta profundo por la impotencia para ser superficial. No irradia deslumbradora luz sino precisa claridad. Servidor fiel de la inteligencia, separa lógicamente, distingue, clasifica y luego junta su labor a la que da genial unidad. Es, por encima de los métodos que le corresponden en su propio campo científico, lo contrario de quienes confunden con vehemencia, de quienes citan sin precisión, de quienes se apuran, de quienes se dispersan. En un plano que cabe llamar filosófico, se debe ensalzar su fundamental eficacia. No lo empequeñecen ni siquiera la aspereza polémica o el egoísmo científico. Este hombre menudo y tímido, con pocos amigos y discípulos, que muchas veces conoció la soledad y a veces el hambre, realizó una de las contribuciones más formidables en el Perú del siglo XX."

La personalidad científica de Augusto Weberbauer, su acercamiento a los alumnos por la enseñanza, la dirección de tesis y trabajos científicos, y debido a la investigacion realizada a nivel nacional, y de calidad interna- 
cional, había sido núcleo para la formación de un grupo de estudiantes distinguidos, entre los que, además de los botánicos ya mencionados, debemos contar a Felipe Ancieta, Aurora Chirinos, Enrique del Solar, luis Gonzales-Mugaburu, María Gómez, Arístides Herrer, Jorge Sánchez, luz Sarmiento y Cristóbal Vecorena.

Así, con esa calidad de Maestro de MaEsTROs, llegó a nosotros la referencia del profesor Dr. Augusto Weberbauer, a quien ya no alcanzamos como profesor regular, pero tuvimos oportunidad de conocerlo como asesor en el laboratorio y en el campo, dirigiendo a sus ex alumnos, que ya eran profesores nuestros.

Es justo mencionar, además, la influencia positiva del profesor de Antropología, el médico Dr. Pedro Weiss, en la orientación de estos destacados jóvenes.

Debemos anotar, también, algo sobre el desarrollo científico y profesional que llegaron a alcanzar esos jóvenes egresados que encontramos cuando llegamos a San Marcos en 1945, y que tuvieron a Weberbauer como su profesor de Botánica y ejemplo de biólogo. Todos ellos, que fueron alumnos estudiosos, inquietos y exigentes consigo mismos, destacaron después como maestros universitarios, como investigadores de nivel internacional, o como los pioneros de áreas del conocimiento biológico de gran auge en nuestros días.

Desde entonces, estamos convencidos de que los estudiantes, won ideas claras y positivas para la institución universitaria, juegan un papel decisivo para su progreso.

Celia Albrizzio Fóntana, especialista en Botánica, además de docente en las Facultades de Ciencias y Educación de San Marcos, logró especial desempeño en la Universidad Nacional de Educación Enrique Guzmán y Valle, La Cantuta.
Clara Canales Geldres ( $\dagger$ ), especialista en Botánica, docente en la Facultad de Ciencias de San Marcos, tuvo especial contribución para mejorar los textos de enseñanza de la Botánica a nivel secundario.

Pedro Coronado Arrascue ( $\dagger$ ), botánico, además de docente en San Marcos en Ciencias y Educación, publicó excelentes textos para la enseñanza de las ciencias biológicas a nivel secundario. Fue Director del Colegio Nacional Nuestra Señora de Guadalupe y Viceministro de Educación.

Ramón Ferreyra Huerta, botánico, profesor de Botánica Sistemática en San Marcos, estudioso, colector incansable. Botánico asesor de la Estación Experimental Agrícola La Molina, Director del Jardín Botánico, Director del Museo de Historia Natural, fundador del Herbario San Marcos en el MHN, Profesor Emérito.

Juana Infantes Vera $(\dagger)$, especialista en Botánica, docente en San Marcos y en colegios, fue enérgica colectora y estudiosa de nuestras plantas andinas y cultora de la etnobotánica. Una de las actividades que inició con su enorme entusiasmo, y no pudo concluir, fue el establecimiento del Jardín Botánico de la Ciudad Universitaria de San Marcos.

LUZ VALCÁRCEL SAFra, especialista en Botánica, docente y decana de la Facultad de Educación de San Marcos, fundadora de la Revista Weberbauer.

Octavio Velarde Núñez (†), botánico, excelente profesor de Botánica General en la Facultad de Ciencias de San Marcos, de Agrostología en Veterinaria; de Botánica General, Botánica Sistemática y Pastos, Director del Jardín Botánico y del Jardín de Pastos en la Universidad Agraria La Molina, donde fue Decano de la Facultad de Agronomía.

Felipe Ancieta Calderón se inició como ayudante de prácticas del curso Zoología Sis- 
temática en San Marcos, a cargo de Don Rufino Aspiazu. Nuestro colega Ancieta, al no recibir apoyo, se retiró de San Marcos y en la selva peruana estudió aspectos de la biología acuática; laboró en la Dirección de Pesca y Caza del Ministerio de Agricultura. Después fue docente a la Universidad de Trujillo, cuya Escuela de Pesquería lleva su nombre, y fue nombrado Doctor Honoris Causa. Fue Director General Científico del Instituto del Mar del Perú, donde actualmente es asesor científico.

Aurora Chirinos Pizarro destacó por excelentes estudios de biología pesquera.Tuvo magnífico desempeño en el Instituto del Mar del Perú y en el Ministerio de Pesquería.

ENRIQue del Solar Cáceda $(\dagger)$, con una innata vocación pesquera, fue impulsor y asesor de la pesquería artesanal e industrial y un sobresaliente asesor del Instituto del Mar del Perú y del Museo de Historia Natural y promotor de los estudios de muchos jóvenes biólogos pesqueros.

María Gómez Calderón destacó en Antropología al lado del profesor PEDRo WEISS, y contribuyó al desarrollo de esta especialidad en la Facultad de Ciencias.

Luis Gonzales-Mugaburu Figueroa, profesor de Zoología General y Zoología Sistemática I en Ciencias y de Parasitología en Veterinaria. Fue el verdadero impulsor de los cambios que ocurrieron en el Departamento de Zoología desde 1946. Profesor Emérito.

Arístides Herrer Alva ( $\dagger$ ) se inició como ayudante de prácticas de Botánica, pero ingresó a un programa internacional de Entomología Médica en el Ministerio de Salud, y destacó en él por sus logros científicos. Fue Jefe del Instituto de Enfermedades Tropicales en Panamá. Sus estudios sobre verruga peruana son notables.
JoRge SÁnchez Romero ( $†$ ), de vocación pesquera, tuvo dignísimo desempeño en la Dirección de Pesca y Caza del Ministerio de Agricultura y como Director Ejecutivo del Instituto del Mar del Perú, incentivando todo tipo de estudios en el ambiente acuático.

Luz Sarmiento Bendezú, la más joven de este grupo, formada al lado de GonzalesMugaburu en Zoología Sistemática I, mantuvo en alto el nivel de la enseñanza y los estudios de los protozoos y los helmintos, formando una eșcuela que se mantiene activa.

Cristóbal Vecorena Linares $(\dagger)$ fue Jefe de Prácticas de Biología General, pero su vocación pesquera lo llevó a una actuación calificada en la Dirección de Pesca y Caza y en el Ministerio de Pesquería.

\section{Establecimiento de EI Día del Biólogo y su significado}

El cumpleaños de Weberbauer era el 26 de noviembre, y el día 27 de noviembre era día feriado no laborable en todo el país. Por tal motivo, el día 27 constituía la oportunidad para agasajar al maestro, lo cual generalmente se hacía con una salida al campo y un almuerzo campestre, con la participación de sus ex alumnos y discípulos, y también se invitaba a otros profesores y a los alumnos de entonces.

El 27 de noviembre de 1947, la reunión se hizo en Surco. Al momento de los discursos, después de la intervención de los participantes mayores, un alumno pidió la palabra y solicitó que:

"El 27 de noviembre se convirtiera en EL DÍA DEL BIÓLOGO, como una ocasión anual para recordar a los maestros, informarnos de los avances y logros en nuestras ciencias, homenajear a los sobresalientes y afianzar nuestra unión y camaradería". 
El pedido fue aprobado, y desde entonces el día central de las conmemoraciones sobre las Ciencias Biológicas es el 27 de noviembre de cada año, a nivel nacional. Dicho alumno de entonces tiene ahora el honor de referirles este hecho, que tuvo un significado muy importante para nuestra profesión.

El 16 de enero de 1948 falleció el Dr. Weberbauer, después de 47 años de permanencia en el Perú y 27 años de servicios a San Marcos. En 1971 el Museo de Historia Natural conmemoró el centenario de su nacimiento.

El 27 de noviembre se convirtió así en la fecha obligada de reunión anual de los biólogos. A los pocos años en una de dichas ocasiones, en San Marcos se fundó la Asociación de Egresados de Ciencias Biológicas de San Marcos, que sirvió para unirnos cada vez más, e incluso para realizar una excursión conjunta al norte del Pení, a Tumbes, con la participación de todos los especialistas sanmarquinos de entonces.

El año 1966 se fundó la Asociación $\mathrm{Na}$ cional de Biólogos del Perú, cuyo presidente fundador fue Luis Gonzales-Mugaburu Figueroa y el vicepresidente, RómULO Jordán SOTELo. Dicha organización recibió delegados de otras universidades.

En 1968, Damisela Coz Campos fue elegida presidenta de la Asociación Nacional de Biólogos, y su actividad fue especialmente distinguida, principalmente porque culminó la gestión de Fundación del Colegio de Biólogos del Perú. Sus colaboradores inmediatos fueron Laura Chevarrí, Secretaria; Dolores neIra, Tesorera; Alberto Cordano, Secretario de Relaciones Públicas.

El Decreto Ley N. ${ }^{\circ} 19364$, del 18 de abril de 1972, creó el Colegio de Biólogos del Perú, a solicitud de la Asociación Nacional de Biólogos del Perú, constituyéndose una Comisión
Especial integrada por trece representantes: uno del Ministerio de Salud, uno del Ministerio de Educación, uno del Consejo Nacional de Investigación, hoy Concytec, uno de la Asociación Nacional de Biólogos, y un representante biólogo de cada una de las universidades estatales y particulares que contaban con Programa Académico de Biología en funcionamiento (fueron Agraria, Arequipa, Ayacucho, Cayetano Heredia, Cuzco, Iquitos, Ricardo Palma, Trujillo y San Marcos).

Dicha Comisión elaboró el Estatuto en el término de 60 días y procedió a la elección del Consejo Nacional, en Lima, y seis Consejos Regionales (DEL Norte, en Trujillo; del CeNtro, en Lima; del Centro Andino, en Ayacucho; DEL SUR, en Arequipa; Del SuR Andino, en Cuzco; de la Amazonia, en Iquitos) en el término de 120 días.

La primera elección de Decano del Consejo Nacional recayó en la Dra. Damisela Coz CAMpos, cuya Directiva juramentó en octubre de 1972, en el local del Colegio de Contadores Públicos de Lima, ante la Comisión Especial constituida por el D. L. 19364, presidida por Pedro Agullar Fernandez.

Se han sucedido los nueve decanos siguientes: Damisela Coz, Enrique Vinatea, Manuel Acosta, Jorge Mejía, Luisa Pilares (todos sanmarquinos), Miguel AguAdo (de la Universidad de Huamanga), Felipe Ancieta (de San Marcos), Isabel Martos (de la Universidad de Trujillo), Magdalena Pavlich (de San Marcos).

Principalmente, primero por acción de la Asociación Nacional de Biólogos del Perú y luego del Colegio de Biólogos del Perú, se han realizado 14 Congresos Nacionales de Biología, en diferentes lugares del Perú: Lima, Piura, Chiclayo, Trujillo, Arequipa, Tacna, Cuzco, Ayacucho, Iquitos. Los trabajos expuestos sólo han sido publicados in extenso en las Memorias del X Congreso realizado en Lima en 1992. 
En los demás casos, sólo algunos trabajos se publicaron dispersos.

Las publicaciones referidas a la Biología son variadas. Cada institución posee alguna forma de divulgación de sus trabajos científicos. La Facultad de Ciencias Biológicas de San Marcos tiene a su cargo la edición de la Revista Peruana de Brología. No olvidemos que lo más importante para publicar es producir investigación de calidad, que merezca su inclusión en revistas bien calificadas.

En el Perú actualmente existen quince Escuelas de Biología: cinco en Lima (Agraria La Molina, Cayetano Heredia, Federico Villarreal, Ricardo Palma y San Marcos), y las otras diez, en las universidades de Arequipa, Ayacucho, Cusco, Ica, Iquitos, Lambayeque, Piura, Puno, Tacna y Trujillo. Sus egresados tienen representantes distinguidos en las diferentes áreas de la Biología; y ellos, por mérito propio, hacen honor a su Alma Máter, prestigiándola y fortaleciéndola.

\section{Palabras finales}

Hemos informado sobre ciertos hechos históricos relacionados con la situación de las Ciencias Biológicas en San Marcos, precedente al establecimiento del Día del Biólogo en el Perú; y brevemente nos hemos referido a los primeros biólogos sanmarquinos de entonces, con destacada gestión y acción para el fortalecimiento de la Biología en el Perú.

En los últimos años, las maestrías y doctorados en Ciencias Biológicas, que se ofrecen en nuestras universidades, deben ser seriamente cimentados y exigentes, para que realmente constituyan una sobresaliente proyección de estas áreas a nivel nacional, y también internacional.

En esta ocasión debemos recordar que los términos de los compromisos que en los últimos años adquieren los biólogos son cada vez más amplios, más serios, más complejos y requieren acción más oportuna, lo cual significa estar mejor preparados, mejor relacionados $y$, fundamentalmente, más unidos como profesión, como la institución que constituimos a nivel nacional, hacia la cual debemos proyectarnos y honrarla con toda sinceridad, probidad y energía al cumplir con nuestras responsabilidades.

No olvidemos que no sólo los biólogos participan en el avance de las ciencias biológicas. Se producen acciones decisivas de médicos humanos, veterinarios, bioquímicos, agrónomos, forestales y de otras profesiones ambientalistas que estudian los seres vivos desde distintos puntos de vista.

No olvidemos tampoco que San Marcos ha sido la cuna de buen número de biólogos pioneros en muchos campos de acción profesional actual, $y$ han sido y siguen siendo maestros en otras universidades de todo el país, nacionales y particulares, en profesiones diferentes.

El reto de continuar y fortalecer y poner al día este desarrollo tiene que ser tomado por profesores y estudiantes de las ciencias biológicas. Todos unidos, y con la visión en el futuro de nuestras instituciones, debemos seguir esforzándonos y venciendo retos, por el bien del Perú.

En más de medio siglo de mi vida como biólogo, ha sido muy grato y tonificante, saber de los progresos de los colegas, de los ex alumnos que ejercen la docencia universitaria sobre la biología moderna, o que son investigadores calificados y en continua superación.

Para conocer el ritmo de los avances de las diferentes áreas de la Biología en el Perú necesitaremos la información que puedan brindar muchos especialistas peruanos que, desde los años 50, es decir, la segunda mitad del siglo $\mathrm{XX}$, fueron pioneros y actualmente pue- 
den ver los resultados de su dedicación. Esta historia, lustro por lustro, o década a década, debe escribirse para aquilatar valores, conocer a los colegas que precedieron o impulsaron el desarrollo actual, $y$ hacer reconocimiento a quien lo merece.
¡Nunca olvidemos que ser Biólogo significa trabajo, dedicación, honestidad, responsabilidad, firmeza, desprendimiento, superación, integración y honor a la verdad! 